\title{
Synthesis and characterization of chloromaleimidobenzenesulfonylhydrazones
}

\author{
Luciano Luiz Silva, Kely Navakoski de Oliveira, and Ricardo José Nunes* \\ Departamento de Química, Universidade Federal de Santa Catarina - UFSC. \\ CEP - 88040-900 - Florianópolis - Santa Catarina - Brazil \\ E-mail:nunes@gmc.ufsc.br
}

\begin{abstract}
This paper describes the synthesis of a new series of imidosulfonylhydrazones in a search for antibactericidal and/or antinociceptive lead compounds.Cyclic imides comprise an important family of organic compounds with therapeutic potential, including the sulfonylhydrazones. 3,4Dichloro-1-phenyl-1H-pyrrole-2,5-dione (1) was obtained from the reaction between aniline and dichloromaleic anhydride in acetic acid. Reaction of (1) with pyrrolidine gave 3-chloro-1phenyl-4-pyrrolidin-1-yl-1H-pyrrole-2,5-dione (2). 4-(3-Chloro-2,5-dioxo-4-pyrrolidin-1-yl-2,5dihydro-1H-pyrrol-1-yl)benzenesulfonyl chloride (3) was obtained from the chlorosulfonation of compound (2). The reaction of (3) with hydrazine hydrate produced 4-(3-chloro-2,5-dioxo-4pyrrolidin-1-yl-2,5-dihydro-1H-pyrrol-1-yl)benzenesulfonohydrazide (4), which was characterized through condensation with aldehydes to yield the imidosulfonylhydrazones (5-12).
\end{abstract}

Keywords: Chloromaleimidobenzenesulfonylhydrazones, sulfonylhydrazones, synthesis

\section{Introduction}

The synthesis of sulfonylhydrazones is of great interest since these compounds have shown antiinflammatory, analgesic ${ }^{1-3}$, anti-pyretic ${ }^{4}$, and antibacterial activities ${ }^{5}$. The antineoplastic activity of some sulfonylhydrazones has also been reported ${ }^{6}$. Studies carried out by Barreiro et al. have shown that sulfonylhydrazone derivatives of safrole have potent analgesic action, exceeding and/or equalling the potency observed under the same conditions for either dypirone or indomethacin ${ }^{7}$. This paper describes the synthesis of chloromaleimidobenzenesulfonylhydrazones, in a search for antibactericidal and/or antinociceptive lead compounds.

\footnotetext{
* to whom correspondence should be addressed
} 


\section{Results and Discussion}

The cyclic imide (1) was obtained from the reaction between aniline and dichloromaleic anhydride with acetic acid under reflux, as previously described ${ }^{8}$ (Scheme 1). Compound (2) was obtained from the reaction of compound (1) $(1 \mathrm{~mol})$ and pyrrolidine $(2 \mathrm{~mol})$ in dichloromethane at room temperature 9 . Compound (3) was prepared by the reaction of compound (2) $(1 \mathrm{~mol})$ with chlorosulfonic acid $(6 \mathrm{~mol})^{9}$. The reaction of the sulfonylchloride with hydrazine hydrate gave the sulfonylhydrazide (4), which was characterized by condensation with aldehydes to yield the sulfonylhydrazones (5-12) (Scheme 1).<smiles>CC(C)(C)OC(=O)C1=C(Cl)C(=O)N(c2ccccc2)C1=O</smiles>

(1)<smiles>O=C1C(Cl)=C(N2CCCC2)C(=O)N1c1ccccc1</smiles>

(2) $\stackrel{\text { eq. } \mathrm{HClSO}_{3}}{\longrightarrow}$<smiles>O=C1C(Cl)=C(N2CCCC2)C(=O)N1c1ccc(S(=O)(=O)Cl)cc1</smiles>

(3)

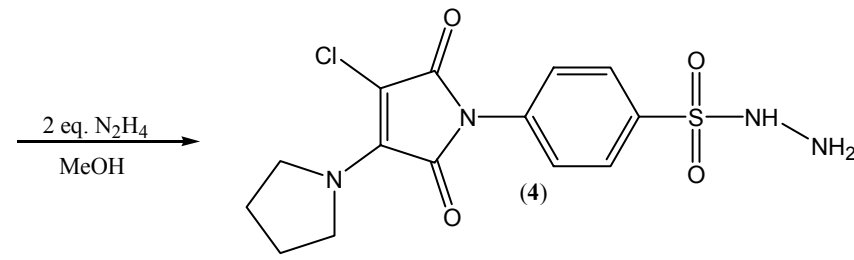

(4)<smiles>[R]C=NNS(=O)(=O)c1ccc(N2C(=O)C(Cl)=C(N3CCCC3)C2=O)cc1</smiles>

$\mathrm{R}=-\mathrm{C}_{6} \mathrm{H}_{5}$

$\mathrm{R}=-\mathrm{C}_{6} \mathrm{H}_{4}-p-\mathrm{OH}(\mathbf{6})$

$\mathrm{R}=-\mathrm{C}_{6} \mathrm{H}_{4}-p-\mathrm{NO}_{2}$ (7)

$\mathrm{R}=-\mathrm{C}_{6} \mathrm{H}_{4}-m-\mathrm{NO}_{2}$ (8)

$\mathrm{R}=-\mathrm{C}_{6} \mathrm{H}_{4}-p-\mathrm{N}\left(\mathrm{CH}_{3}\right)_{2}$ (9)

$\mathrm{R}=\overbrace{\mathrm{O}}^{\mathrm{O}} \mathrm{C}(10)$

$\mathrm{R}=\longrightarrow \square^{\mathrm{O}} \rrbracket(11)$

$\mathrm{R}=\mathbb{U}^{\mathrm{S}}{ }_{(12)}$

\section{Scheme 1}


The configuration of the imino double bonds of (5-12) could not be determined by NMR data, where only one imino hydrogen was observed. However, a study of the relative stability of the $E / Z$ diastereomers involved, employing the Hamiltonian PM3 molecular model and the work of Barreiro et al. ${ }^{10}$, indicated that the $E$ isomers may be preferentially formed. The structures were confirmed through ${ }^{1} \mathrm{H}$ NMR and ${ }^{13} \mathrm{C}$ NMR spectroscopic analysis and $\mathrm{CNH}$ elemental analysis.

\section{Experimental Section}

General Procedures. All compounds were characterised by ${ }^{1} \mathrm{H}$ NMR, ${ }^{13} \mathrm{C} N M R$, IR, and microanalysis. The purity of these compounds was determined by thin layer chromatography (TLC). Infrared spectra were obtained with a Perkin Elmer 16PC spectrophotometer (Perkin Elmer, Wellesley, MA, USA). ${ }^{1} \mathrm{H}$ NMR and ${ }^{13} \mathrm{C}$ NMR spectra were recorded with a Bruker AC200F spectrometer (Rheinstetten, Germany) (at $200 \mathrm{MHz}$ and $50 \mathrm{MHz}$, respectively). $\mathrm{CDCl}_{3}$ and DMSO were used as solvents with tetramethylsilane (TMS) as the internal standard; chemical shifts $(\delta)$ are reported in parts per million. For the CHN analysis, a CHN elemental analyser PERKIN ELMER 2400 (Boston, MA, USA) was used. In the TLC, aluminium sheets with 60 F254 silica gel and $0.2 \mathrm{~mm}$ thickness were utilised.

3,4-Dichloro-1-phenyl-1H-pyrrole-2,5-dione (1). Imide (1) was obtained as describe in the literature ${ }^{8}$. Yield: $82 \%$. mp 206-207 ${ }^{\circ} \mathrm{C}$ ( Lit $^{8}$ m.p. $\left.204-206^{\circ} \mathrm{C}\right)$.

3-Chloro-1-phenyl-4-pyrrolidin-1-yl-1H-pyrrole-2,5-dione (2). Imide (2) was obtained as describe in the literature ${ }^{9}$. Yield: $80 \%$ mp $134-135^{\circ} \mathrm{C}\left(\mathrm{Lit}^{9}\right.$ m.p. $\left.135-136^{\circ} \mathrm{C}\right)$.

4-(3-Chloro-2,5-dioxo-4-pyrrolidin-1-yl-2,5-dihydro-1H-pyrrol-1-yl)benzenesulfonyl

chloride (3). Chloride (3) was obtained as described in the literature ${ }^{9}$. Yield: 89 \%. mp 121-123 ${ }^{\circ} \mathrm{C}$ (Lit ${ }^{9}$ m.p. $\left.122-123^{\circ} \mathrm{C}\right)$.

\section{4-(3-Chloro-2,5-dioxo-4-pyrrolidin-1-yl-2,5-dihydro-1 $H$-pyrrol-1-yl)benzenesulfono}

hydrazide (4). Hydrazine hydrate $(51.3 \mathrm{mg}, 1.60 \mathrm{mmol})$ was added to a mixture of 4-(3-chloro2,5-dioxo-4-pyrrolidin-1-yl-2,5-dihydro-1H-pyrrol-1-yl)benzenesulfonyl chloride (3) (0.3 g, 0.80 mmol) in methanol $(30 \mathrm{~mL})$ at $0{ }^{\circ} \mathrm{C}$. The mixture was allowed to warm to room temperature for 30 minutes and was then poured onto ice-water. The solid formed was obtained through filtration with suction. The product was crystallized from hexane-chloroform (1:2). Yield: $89 \%$ mp 178.1-179.0 ${ }^{\circ} \mathrm{C} . \operatorname{IR}(\mathrm{KBr}) v_{\mathrm{Max}}: 3383,3192,2963,1777,1708,1387,1193,1173,837$.

\section{4-(3-Chloro-2,5-dioxo-4-pyrrolidin-1-yl-2,5-dihydro-1H-pyrrol-1-yl)- $N$ '-[(1E)phenyl-}

methylene]benzenesulfonohydrazide (5). Benzaldehyde $(28.6 \mathrm{mg}, 0.27 \mathrm{mmol})$ was added to a mixture of 4-(3-chloro-2,5-dioxo-4-pyrrolidin-1-yl-2,5-dihydro-1H-pyrrol-1yl)benzenesulfono hydrazide (4) $(0.10 \mathrm{~g}, 0.27 \mathrm{mmol})$ in ethanol $(10 \mathrm{~mL})$, along with a drop of hydrochloric acid as the catalyst. The reaction was left under stirring at room temperature for $1 \frac{1 / 2}{2}$ hours. The solid formed was obtained through filtration with suction. The product was crystallized from ethanol-ethyl acetate $(2: 1)$ as light yellow colored crystals. Yield: $73 \%$ mp 
189.2-190.5 ${ }^{\circ} \mathrm{C} . \operatorname{IR}(\mathrm{KBr}) v_{\operatorname{Max}}$ 3210, 2970, 1770, 1704, 1635, 1493, 1386, 1167, 1232. Anal. Calcd. for $\mathrm{C}_{21} \mathrm{H}_{19} \mathrm{Cl} \mathrm{N}_{4} \mathrm{O}_{4} \mathrm{~S}$ : C, 54.96; H, 4.17; Cl, 7.73; N, 12.21; S, 6.99. Found: C, 54.49; H, 4.30; N, 12.10; S, 6.94. ${ }^{1} \mathrm{H}$ NMR $\delta$ ppm, DMSO- $d^{6}: 11.58$ (s, $\left.1 \mathrm{H}:-\mathrm{NH}-\mathrm{N}=\right)$ ); 7.98 and $7.94(2 \mathrm{~d}$, 2H: $\operatorname{ArH~J}=8.2 \mathrm{~Hz}$ ); 7.93 (s, 1H: $-\mathrm{N}=\mathrm{CH}-) 7.55$ (m, 2H: $\mathrm{ArH}) ; 7.40$ (m, 5H: ArH); 3.88- (s, 4H: $\left.\mathrm{CH}_{2}-\mathrm{N}-\mathrm{CH}_{2}\right) ; 1.87$ (s, 4H: $\left.\mathrm{CH}_{2}-\mathrm{CH}_{2}\right) .{ }^{13} \mathrm{C} \mathrm{NMR} \delta \mathrm{ppm}, \mathrm{DMSO}-d^{6}: 25.31\left(\underline{\mathrm{CH}}_{2}-\underline{\mathrm{CH}}_{2}-\mathrm{CH}_{2}-\mathrm{N}\right)$; 51.11 $\left(\mathrm{CH}_{2}-\mathrm{N}-\mathrm{CH}_{2-}\right) ; 89.87(\mathrm{Cl}-\mathrm{C}=\mathrm{C}) ; 127.23,127.54 ; 128.57 ; 129.50 ; 130.88 ; 134.25(\mathrm{CH} \mathrm{Ar})$; 136.39 (-Cl-C=-ㅡ); 137.81 (-C Ar-SO ${ }_{2}$ ); 141.72 (-C Ar-N-), 148.20 (-N=C-); 163.45 (C=O), $165.57(\mathrm{C}=\mathrm{O})$.

Using this procedure the following sulfonylhydrazones were prepared:

4-(3-Chloro-2,5-dioxo-4-pyrrolidin-1-yl-2,5-dihydro-1H-pyrrol-1-yl)- $N^{\prime}-[(1 E)-(4-h y d r o x y-$ phenyl)methylene]benzenesulfonohydrazide (6). Yield: $71 \%$. Dec.: $200{ }^{\circ} \mathrm{C}$. $I R(\mathrm{KBr}) v_{\operatorname{Max}}$ : 3452 , 3156, 2995, 1759, 1706, 1626, 1499, 1389, 1174, 1232. Anal. Calcd. for $\mathrm{C}_{21} \mathrm{H}_{19} \mathrm{Cl} \mathrm{N}_{4} \mathrm{O}_{5}$ S: C, 53.11; H, 4.03; Cl, 7.47; N, 11.80; S, 6.75. Found: C, 52.87; H, 4.18; N, 11.74; S, 6.71. ${ }^{1} \mathrm{H}$ NMR $\delta$ ppm, DMSO- $d^{6}: 11.27$ (s, 1H: -NH-N=); 9.91 (s, 1H: OH); 7.95 and 7.91 (2d, 2H: ArH; $\mathrm{J}=8.0 \mathrm{~Hz}$ ); 7.82 (s, 1H: $-\mathrm{N}=\mathrm{CH}-) ; 7.60$ and $7.56(2 \mathrm{~d}, 2 \mathrm{H}: \mathrm{ArH} ; \mathrm{J}=8.0 \mathrm{~Hz}) ; 7.42$ and 7.38 (2d, 2H: $\mathrm{ArH} ; \mathrm{J}=8.0 \mathrm{~Hz}) ; 6.78$ and $6.74(2 \mathrm{~d}, 2 \mathrm{H}: \mathrm{ArH} ; \mathrm{J}=8.0 \mathrm{~Hz}) ; 3.88\left(\mathrm{~s}, 4 \mathrm{H}:-\mathrm{CH}_{2}-\mathrm{N}-\mathrm{CH}_{2}-\right) ; 1.87$ (s, 4H: $\left.-\mathrm{CH}_{2}-\mathrm{CH}_{2}-\right) .{ }^{13} \mathrm{C}$ NMR $\delta$ ppm, DMSO- $d^{6}: 24.70\left(\mathrm{CH}_{2}-\mathrm{CH}_{2}-\mathrm{CH}_{2}-\mathrm{N}\right) ; 50.51\left(\mathrm{CH}_{2}-\mathrm{N}-\mathrm{CH}_{2}-\right.$ ); $109.26(\mathrm{Cl}-\underline{\mathrm{C}}=\mathrm{C}) ; 115.71 ; 124.66 ; 126.61 ; 127.96 ; 128.72$ ( $\mathrm{CH} \mathrm{Ar}) ; 135.65$ (-Cl-C=- ); $137.29\left(-\mathrm{C} \mathrm{Ar}-\mathrm{SO}_{2}\right) ; 141.12(-\mathrm{C} \mathrm{Ar}-\mathrm{N}) ; \quad 148.14(-\mathrm{N}=\mathrm{C}-) ; 159.51(-\mathrm{HC}-\mathrm{OH} \mathrm{Ar}) ; 162.86(\mathrm{C}=\mathrm{O})$; $165.00(\mathrm{C}=\mathrm{O})$.

4-(3-Chloro-2,5-dioxo-4-pyrrolidin-1-yl-2,5-dihydro-1 $H$-pyrrol-1-yl)- $N^{\prime}-[(1 E)-(4-$ nitrophenyl)methylene]benzenesulfonohydrazide (7). Yield: $80 \%$. mp 201.0-202.7 ${ }^{\circ} \mathrm{C}$. $I R(\mathrm{KBr}) v_{\text {Max }}$ : 3240, 2990, 1759, 1706, 1630, 1495, 1522, 1348, 1385, 1168, 1226, 852. Anal. Calcd. for $\mathrm{C}_{21} \mathrm{H}_{18} \mathrm{Cl} \mathrm{N}_{5} \mathrm{O}_{6} \mathrm{~S}$ : C, 50.05; H, 3.60; Cl, 7.04; N, 13.90; S, 6.36. Found: C, 49.76; H, 3.83; N, 13.85; S, 6.22. ${ }^{1} \mathrm{H}$ NMR $\delta$ ppm, DMSO- $d^{6}: 11.96(\mathrm{~s}, 1 \mathrm{H}:-\mathrm{NH}-\mathrm{N}=) ; 8.26$ and $8.22(2 \mathrm{~d}$, 2H: $\mathrm{ArH}, \mathrm{J}=8.2 \mathrm{~Hz}$ ); 7.99 (s, 1H: -N=CH-) 7.96 (m, 4H: ArH); 7.59 (m, 2H: ArH); 3.89 (s, 4H: $\left.-\mathrm{CH}_{2}-\mathrm{N}-\mathrm{CH}_{2}-\right) ; 1.86\left(\mathrm{~s}, 4 \mathrm{H}:-\mathrm{CH}_{2}-\mathrm{CH}_{2}-\right) .{ }^{13} \mathrm{C}$ NMR $\delta$ ppm, DMSO- $d^{6}: 25.32\left(\underline{\mathrm{CH}_{2}-} \underline{\mathrm{CH}}_{2}-\mathrm{CH}_{2}-\mathrm{N}\right)$; $51.11\left(\mathrm{CH}_{2}-\mathrm{N}-\mathrm{CH}_{2}-\right) ; 85.56(\mathrm{Cl}-\underline{\mathrm{C}}=\mathrm{C}) ; 124.73,127.33 ; 128.53$ (CH Ar); 136.56 (-Cl-C=-ㅡ); $140.42\left(-\mathrm{C} \mathrm{Ar}-\mathrm{SO}_{2}\right) ; 141.75$ (-C Ar-N); 145.60- (CH-NO $\left.{ }_{2} \mathrm{Ar}\right), 148.60$ (-N=C-); $163.43(\mathrm{C}=\mathrm{O})$; $165.54(\mathrm{C}=\mathrm{O})$.

4-(3-Chloro-2,5-dioxo-4-pyrrolidin-1-yl-2,5-dihydro-1 $H$-pyrrol-1-yl)- $N$ '-[(1E)-(3-

nitrophenyl)methylene]benzenesulfonohydrazide (8). Yield: 88 \%. mp 199.6-201.4 ${ }^{\circ} \mathrm{C}$. $I R(\mathrm{KBr}) v_{\text {Max }}$ : 3208, 2985, 1764, 1706, 1633, 1500, 1543, 1348, 1372, 1166, 1241, 869. Anal. Calcd. for $\mathrm{C}_{21} \mathrm{H}_{18} \mathrm{Cl} \mathrm{N}_{5} \mathrm{O}_{6} \mathrm{~S}$ : C, 50.05; H, 3.60; Cl, 7.04; N, 13.90; S, 6.36. Found: C, 49.96; H, 3.74; N, 13.77; S, 6.24. ${ }^{1} \mathrm{H}$ NMR $\delta$ ppm, DMSO- $d^{6}: 11.97$ (s, 1H: -NH-N=); 8.38 (m, 2H: ArH); 8.24 and 8.20 (2d, 2H: $\mathrm{ArH}, \mathrm{J}=8.0 \mathrm{~Hz}) ; 8.15-(\mathrm{s}, 1 \mathrm{H}: \mathrm{N}=\mathrm{CH}-) 8.04$ (m, 2H: $\mathrm{ArH}) ; 7.61$ (2d, 2H: $\operatorname{ArH~J}=8,1) ; 3.90$ (s, 4H: $\left.-\mathrm{CH}_{2}-\mathrm{N}_{-} \mathrm{CH}_{2}-\right) ; 1.87$ (s, 4H: $\left.-\mathrm{CH}_{2}-\mathrm{CH}_{2}-\right) .{ }^{13} \mathrm{C} \mathrm{NMR} \delta$ ppm, DMSO- $d^{6}$ : $25.30\left(\underline{\mathrm{CH}}_{2}-\underline{\mathrm{CH}}_{2}-\mathrm{CH}_{2}-\mathrm{N}\right) ; 51.11 \quad\left(\mathrm{CH}_{2}-\mathrm{N}-\mathrm{CH}_{2}-\right) ; 88.98 \quad(\mathrm{Cl}-\underline{\mathrm{C}}=\mathrm{C}) ; 121.91,125.12 ; 127.30$, 128.53, 131.13, 133.41, 136.05 ( $\mathrm{CH} \mathrm{Ar}) ; 136.52$ (-Cl-C=-ㅡ); 137.70 (-C Ar-SO 2$) ; 141.72(-\mathrm{C}$ Ar-N); 145.84 (-C Ar-NO $), 148.84(-\mathrm{N}=\mathrm{C}-) ; 163.43(\mathrm{C}=\mathrm{O}), 165.70(\mathrm{C}=\mathrm{O})$. 
4-(3-Chloro-2,5-dioxo-4-pyrrolidin-1-yl-2,5-dihydro-1 $H$-pyrrol-1-yl)- $\boldsymbol{N}^{\prime}-\{(1 E)-[4-$

(dimethylamino)phenyl]methylene\}benzenesulfonohydrazide (9). $\quad$ Yield: $71 \%$. Dec. $170{ }^{\circ} \mathrm{C} . I R(\mathrm{KBr})$ $v_{\text {Max }}: 3126,2978,1770,1717,1634,1495,1365,1163,1232$.Anal. Calcd. for $\mathrm{C}_{23} \mathrm{H}_{24} \mathrm{Cl} \mathrm{N}_{5} \mathrm{O}_{4} \mathrm{~S}$ : C, 55.03; H, 4.82; Cl, 7.06; N, 13.95; S, 6.39. Found: C, 54.83; H, 4.98; N, 13.82; S, 6.26. ${ }^{1} \mathrm{H}$ NMR $\delta$ ppm, DMSO- $d^{6}: 11.32$ (s, 1H: $\left.-\mathrm{NH}-\mathrm{N}=\right)$; 7.97 and 7.93 (2d, 2H: ArH, J=8.0 Hz); 7.83 (s, 1H: $\mathrm{N}=\mathrm{CH}-) ; 7.59$ and 7.55 (2d, 2H: $\mathrm{ArH}, \mathrm{J}=8.0 \mathrm{~Hz}) ; 7.45$ and $7.41(2 \mathrm{~d}, 2 \mathrm{H}: \mathrm{ArH}, \mathrm{J}=8.0 \mathrm{~Hz}) ; 6.91$ and $6.87(2 \mathrm{~d}, 2 \mathrm{H}$ : $\mathrm{ArH}, \mathrm{J}=8.0 \mathrm{~Hz}) ; 3.89\left(\mathrm{~s}, 4 \mathrm{H}:-\mathrm{CH}_{2}-\mathrm{N}-\mathrm{CH}_{2}-\right) ; 2.97\left(\mathrm{~s}, 6 \mathrm{H}:-\mathrm{N}-\left(\mathrm{CH}_{3}\right)_{2}\right) ; 1.88(\mathrm{~s}$, $\left.4 \mathrm{H}-\mathrm{CH}_{2}-\mathrm{CH}_{2}-:\right) .{ }^{13} \mathrm{C}$ NMR $\delta$ ppm, DMSO- $d^{6}: 24.68\left(\mathrm{CH}_{2}-\underline{C H}_{2}-\mathrm{CH}_{2}-\mathrm{N}\right) ; 41.13\left(-\mathrm{N}-\left(\mathrm{CH}_{3}\right)_{2}\right)$; $50.47\left(\mathrm{CH}_{2}-\mathrm{N}-\mathrm{CH}_{2}-\right) ; 110.53(\mathrm{Cl}-\underline{\mathrm{C}}=\mathrm{C}) ; 114.09,126.55,127.95,128.26(\mathrm{CH} \mathrm{Ar}) ; 135.61$ (-Cl$\mathrm{C}=\underline{\mathrm{C}}-) ; 137.29\left(-\mathrm{C} \mathrm{Ar}-\mathrm{SO}_{2}\right) ; 141.07(-\mathrm{C} \mathrm{Ar}-\mathrm{N}) ; 148.09\left(-\mathrm{CH}-\mathrm{N}\left(\mathrm{CH}_{3}\right)_{2}\right), 149.86(-\mathrm{N}=\mathrm{C}-) ; 162.81$ $(\mathrm{C}=\mathrm{O}), 164.96(\mathrm{C}=\mathrm{O})$.

\section{4-(3-Chloro-2,5-dioxo-4-pyrrolidin-1-yl-2,5-dihydro-1 H-pyrrol-1-yl)- $N$-[(1E)-1,3-benzodioxol-5-}

ylmethylene]benzenesulfonohydrazide (10). Yield: $69 \%$. mp $217.5-218.1^{\circ} \mathrm{C} . I R(\mathrm{KBr}) v_{\text {Max: }}$ 3157, 2976, 1764, 1706, 1632, 1500, 1372, 1163, 1258. Anal. Calcd. for $\mathrm{C}_{22} \mathrm{H}_{19} \mathrm{Cl} \mathrm{N}_{4} \mathrm{O}_{6} \mathrm{~S}: \mathrm{C}, 52.54 ; \mathrm{H}, 3.81 ; \mathrm{Cl}, 7.05 ; \mathrm{N}$, 11.14; S, 6.38. Found: C, 52.25; H, 3.90; N, 11.03; S, 6.15. ${ }^{1} \mathrm{H}$ NMR $\delta$ ppm, DMSO- $d^{6}$ : 11.36 (s, 1H: $-\mathrm{NH}-\mathrm{N}=)$; 7.98 and 7.94 (2d, 2H: $\mathrm{ArH}, \mathrm{J}=8.4 \mathrm{~Hz}) ; 7.83$ (s, 1H: $-\mathrm{N}=\mathrm{CH}-) ; 7.61$ and 7.57 (2d, 2H: $\mathrm{ArH}, \mathrm{J}=8.4 \mathrm{~Hz}$ ); 7.03 (m, 3H: $\mathrm{ArH}) ; 6.05$ (s, 2H: O-CH$\left.{ }_{2}-\mathrm{O}\right) ; 3.90$ (s, 4H: $-\mathrm{CH}_{2}-\mathrm{N}_{-}-\mathrm{CH}_{2}-$ ); 1.89 (s, 4H: $\left.-\mathrm{CH}_{2}-\mathrm{CH}_{2}-\right) .{ }^{13} \mathrm{C}$ NMR $\delta$ ppm, DMSO- $d^{6}: 24.68\left(\mathrm{CH}_{2}-\mathrm{CH}_{2}-\mathrm{CH}_{2}-\mathrm{N}\right) ; 50.47\left(\mathrm{CH}_{2}-\mathrm{N}-\right.$ $\left.\mathrm{CH}_{2}-\right) ; 101.60\left(\mathrm{O}-\mathrm{CH}_{2}-\mathrm{O}\right) ; 104.98(\mathrm{Cl}-\underline{\mathrm{C}}=\mathrm{C}) ; 108.45,123.24,126.58,127.97,147.45,147.99$ $\left(\mathrm{CH}\right.$ Ar); $135.70(-\mathrm{Cl}-\mathrm{C}=\underline{\mathrm{C}}) ; 137.11\left(-\mathrm{C} \mathrm{Ar}-\mathrm{SO}_{2}\right) ; 141.08$ (-C Ar-N);149.18 (-N=C-);162.81 $(\mathrm{C}=\mathrm{O}) ; 164,96(\mathrm{C}=\mathrm{O})$.

4-(3-Chloro-2,5-dioxo-4-pyrrolidin-1-yl-2,5-dihydro-1 $H$-pyrrol-1-yl)- $N$ '-[(1E)-2-

furylmethylene]benzenesulfonohydrazide (11). Yield: $64 \%$. Dec.: $195.0^{\circ} \mathrm{C} . \operatorname{IR}(\mathrm{KBr}) v_{\operatorname{Max}}$ : 3147, 2995, 1759, 1701, 1638, 1506, 1378, 1168, 1240. Anal. Calcd. for $\mathrm{C}_{19} \mathrm{H}_{17} \mathrm{Cl} \mathrm{N}_{4} \mathrm{O}_{5} \mathrm{~S}$ : C, 50.84; H, 3.82; Cl, 7.90; N, 12.48; S, 7.14. Found: C, 50.67; H, 3.86; N, 12.43; S, 7.01. ${ }^{1} \mathrm{H}$ NMR $\delta$ ppm, DMSO-d $d^{6}: 11.57$ (s, 1H: $\left.-\mathrm{NH}-\mathrm{N}=\right) ; 7.95$ (s, 1H: -N=CH-) 7.85 (m, 2H: ArH); 7.60 (m,

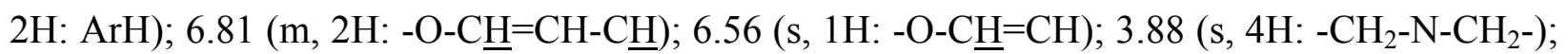
1.87 (s, 4H: $\left.-\mathrm{CH}_{2}-\mathrm{CH}_{2}-\right) .{ }^{13} \mathrm{C} \mathrm{NMR} \delta \mathrm{ppm}, \mathrm{DMSO}-d^{6}: 28.73\left(\underline{\mathrm{CH}}_{2}-\underline{\mathrm{CH}}_{2}-\mathrm{CH}_{2}-\mathrm{N}\right) ; 54.55\left(\mathrm{CH}_{2}-\mathrm{N}-\right.$ $\left.\mathrm{CH}_{2}-\right) ; 109.33(\mathrm{Cl}-\underline{\mathrm{C}}=\mathrm{C}) ; 113.54(-\mathrm{O}-\mathrm{CH}=\underline{\mathrm{CH}}-\mathrm{CH}), 125.32(-\mathrm{O}-\mathrm{CH}=\mathrm{CH}-\underline{\mathrm{CH}}), 130.68 ; 131.95$; 135.55 (CH Ar); 133.05 (-Cl-C=C- $)$; 137.17 (-C Ar-N- and $-\mathrm{O}-\underline{\mathrm{CH}}=\mathrm{CH}-\mathrm{CH}) ; 145.19(-\mathrm{N}=\mathrm{C}-$ and $\mathrm{HC}=\mathrm{C}-\mathrm{O}-) ; 163.75(\mathrm{C}=\mathrm{O}) ; 165.96(\mathrm{C}=\mathrm{O})$.

4-(3-Chloro-2,5-dioxo-4-pyrrolidin-1-yl-2,5-dihydro-1H-pyrrol-1-yl)- $N$ '-[(1E)-thien-2ylmethylene]benzenesulfonohydrazide (12). Yield: $66 \%$. Dec. $209{ }^{\circ} \mathrm{C}$. $I R(\mathrm{KBr}) v_{\mathrm{Max}}: 3152$, 2986, 1759, 1706, 1631, 1506, 1387, 1168, 1233. Anal. Calcd. for $\mathrm{C}_{19} \mathrm{H}_{17} \mathrm{Cl} \mathrm{N}_{4} \mathrm{O}_{4} \mathrm{~S}_{2}$ : C, 49.08; H, 3.69; Cl, 7.63; N, 12.05; S, 13.79. Found: C, 48.97; H, 3.89; N, 11.98; S, 13.66. ${ }^{1} \mathrm{H}$ NMR $\delta$ ppm, DMSO-d $d^{6}: 11.52$ (s, 1H: -NH-N=); 8.12 (s, 1H: -N=CH-); 7.94 and 7.90 (2d, 2H: ArH, J= $8.0 \mathrm{~Hz}$ ); 7.62 and $7.57(2 \mathrm{~d}, 2 \mathrm{H}: \mathrm{ArH}, \mathrm{J}=8.0 \mathrm{~Hz}) ; 7.20(\mathrm{~m}, 3 \mathrm{H}:-\mathrm{S}-\mathrm{C} \underline{\mathrm{H}}=\mathrm{C} \underline{\mathrm{H}}-\mathrm{C} \underline{\mathrm{H}}) ; 3.89$ (s, 4H: $\left.\mathrm{CH}_{2}-\mathrm{N}-\mathrm{CH}_{2}-\right) ; 1.88$ (s, 4H: $\left.-\mathrm{CH}_{2}-\mathrm{CH}_{2}-\right) .{ }^{13} \mathrm{C} \mathrm{NMR} \delta$ ppm, DMSO- $d^{6}: 24.69\left(\underline{\mathrm{CH}}_{2}-\underline{\mathrm{CH}}_{2}-\mathrm{CH}_{2}-\mathrm{N}\right)$; $50.50\left(\mathrm{CH}_{2}-\mathrm{N}-\mathrm{CH}_{2}-\right) ; 112.12(\mathrm{Cl}-\underline{\mathrm{CH}}=\mathrm{CH}) ; 114.34 ; 120.12 ; 126.72 ; 127.58(\mathrm{CH} \mathrm{Ar}) ; 127.90$ (- 


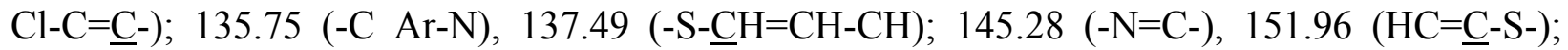
$161.89(\mathrm{C}=\mathrm{O}) ; 163.91(\mathrm{C}=\mathrm{O})$.

\section{Acknowledgements}

The authors are grateful to CNPq for financial support of this work.

\section{References}

1. Sondhi, S. M.; Dinodia, M.; Kumar, A. Bioorg. Med. Chem. 2006, 14, 4657.

2. Cunha, A C.; Tributino, J. L. M.; Miranda, A L. P.; Fraga, C. A M.; Barreiro, E. J. Il Fármaco 2002, 57, 999.

3. Murineddu, G.; Loriga, G.; Gavini, E.; Peana, A T.; Mule, A C.; Pinna, G. A. Arch. Pharm. 2002, 334, 393.

4. Sridar, S. K.; Ramesh, A. Biol. Pharm. Bull. 2001, 24, 1149.

5. Kalluraya, B.; Isloor, A M.; Frank, P. V.; Jagadeesha, R. L. Ind. J. Het. Chem. 2004, 13, 245.

6. Loh, W.; Cosby, L. A.; Sartorelli, A C. J. Med. Chem. 1980, 23, 631.

7. Lima, L.M.; Amarante, E.G.; Miranda, A.L.P.; Fraga, C.A.M.; Barreiro, E.J., Pharm. Pharmacol. Commun. 1999, 5, 673.

8. Martin, E. L. and Dickson, C. L. J.Org. Chem. 1961, 26, 2032.

9. Walter, M. E.; Mora, C.; Mundstock, K.; Souza, M. M.; Pinheiro, A. O.; Yunes, R. A.; Nunes, R. J. Arch. Pharm. Pharm. Med. Chem. 2004, 337, 201.

10. Lima, P. C.; Lima, L. M.; Da Silva, K. C. M.; Leda, P. H. O.; De Miranda, A. L. P.; Fraga, C. A. M.; Barreiro, E. J. Eur. J. Med. Chem. 2000, 35, 187. 